Digital Press Social Sciences and Humanities

Le conflit intérieur du personnage principal dans l'œuvre cinématographique « Respire » de Mélanie Laurent (2014)

Flavera Voni Titanka, Lina Syawalina and Dian Agustina Pratama

Proceeding of Conférence internationale sur le français 2018

Joesana Tjahjani, Merry Andriani, Sajarwa, Wening Udasmoro (eds) 


\title{
Le conflit intérieur du personnage principal dans l'œuvre cinématographique Respire de Mélanie Laurent (2014)
}

\author{
Flavera Voni Titanka, Lina Syawalina, et Dian Agustina Pratama \\ Sekolah Tinggi Bahasa Asing Yapari-ABA Bandung, Bandung, Indonesia \\ *e-mail : flavera.titanka@gmail.com
}

\section{Résumé}

Ce mémoire vise à identifier les formes du conflit intérieur du personnage principal de l'œuvre cinématographique « Respire ». L'objet de cette recherche est le conflit intérieur vécu par le personnage principal dans le film "Respire », réalisé par Mélanie Laurent en 2014. La méthode de recherche est descriptive-qualitative. Le réassemblage des données est fait par la méthode de regarder le film, la prise de note ainsi que l'étude bibliographique. Les données obtenues sont analysées en utilisant la théorie d'humaniste sur motivation et personnalité. Le résultat de cette recherche montre que le conflit intérieur du personnage principal est causé par l'insatisfaction de besoin de sécurité, de besoin d'amour et d'appartenance ainsi que l'insatisfaction de besoin d'estime de soi ce qui donnent les effets psychologiques comme le besoin névrotique.

\section{Mots-clés}

psychologie littéraire, besoin des humains, motivation et personnalité

\begin{abstract}
This research aims to identify the forms of the internal conflict of the main character of the cinematographic work «Respire». The object of this research is the internal conflict experienced by the main character in the film «Respire», directed by Mélanie Laurent in 2014. The research method is descriptive-qualitative. The collection of data is done by the method of watching the film, the note taking as well as the bibliographic study. The data obtained are analysed using humanist theory on motivation and personality. The result of this research shows that the inner conflict of the main character is caused by dissatisfaction with the need for security, need for love and belonging as well as dissatisfaction with the need for self-esteem which give the psychological effects as the neurotic need.
\end{abstract}

\section{Keywords}

literary psychology, human need, motivation and personality

\section{Introduction}

Madame Bovary, Les Misérables sont les films adaptés d'un roman. Aujourd'hui, il est possible de présenter les idées, l'avis, ou les œuvres en utilisant les médias visuels. De cette manière, on peut jouir des œuvres à la façon plus vivante. Nous pouvons dire qu'un film est aussi l'œuvre littéraire imaginative qui est visualisée. En utilisant le film, l'écrivain et aussi le metteur en scène qui peut déterminer le sentiment des spectateurs en représentant le personnage, l'intrigue, et le conflit. Une grande variété de formes de conflit présenté par le scénariste, dont l'un est une forme de harcèlement scolaire. Il existe beaucoup de films français ayant un thème sur le harcèlement comme "L'école m'a tué », "Le mur de l'humiliation », et « Matilda ».

En France, le phénomène de harcèlement est vraiment inquiétant, cité sur le site internet Le Monde.fr (Battaglia, 2017), les statistiques ministérielles déclarent qu'il existe 700000 jeunes possédant 
l'expérience du harcèlement. D'habitude, les garçons sont harcelés physiquement, quant aux filles sont plus exposées au cyber harcèlement, en particulier au collège. Selon les données existant sur le site internet ci-dessus, le pourcentage des élèves à l'école primaire reçoivent le plus traitement de harceler. Il est certain que ces actions inappropriées peuvent même être expérimentées aux enfants de l'école primaire. D'habitude cette action de harcèlement à la victime de façon isolée et aussi les agressions de plusieurs natures « verbale, physique, etc ». Ce phénomène est présenté dans le film « Respire », un film qui raconte l'histoire d'une personne adolescente de lycée comme le personnage principal qui a les problèmes psychologiques. Le film "Respire » est adapté d'un roman avec le même titre, écrit pas Anne Sophie Brasme et a été traduit aux 35 différents langues. Ce film aussi a été nominé comme le film avec le meilleur narratif par les trois différentes Awards, c'est-à-dire Hamptons International Film Festival (2014), Provincetown International Film Festival (2015) et Atlanta Film Festival (2015).

Les aspects psychologiques du film reflètent les conflits exprimés par le personnage principal comme un adolescent qui vient d'une famille qui n'était pas harmonieuse, puis elle a rencontré un nouvel élève dans son école qui garde son secret. Puisque le personnage principal découvre le secret de son ami, elle était harcelée et terrorisée tous les jours. Le personnage principal ou la victime, placée dans une situation pressée soit mentalement soit psychologiquement qui a donné l'impact psychologique. L'existence d'action inappropriée par les amis du personnage principal conduise à un sentiment d'être harcelé et pas apprécié. Le personnage principal se sentait également placé dans une position où elle n'a pas d'amis, cela provoque le stress de personnage principal, et a causé l'apparition du conflit intérieur dans son caractère.

Le grand nombre de problèmes présentés a rendu le film concernant des aspects psychologiques. Ainsi, pour vérifier ces aspects psychologiques, nous révisons et décrivons le scénario dans le film « Respire », particulièrement les dialogues qui décrivent le conflit intérieur du personnage principal. Cette recherche utilise une approche de la psychologie littéraire, cela veut dire une étude créative et interdisciplinaire. La théorie que nous avons utilisée est la théorie d'humaniste sur motivation et personnalité. Nous espérons que cette recherche sera utile pour le développement d'étude de la littérature et aussi pour le cours de la psychologie littéraire en particulier pour les étudiants du département de la littérature. Il est également prié que les lecteurs puissent comprendre les aspects psychologiques contenus dans les œuvres littéraires, notamment dans le film « Respire » de Mélanie Laurent. Cette recherche a pour but de décrire les formes du conflit intérieur du personnage principal de l'œuvre cinématographique « Respire » de Mélanie Laurent.

\section{Discussion}

\subsection{Le harcèlement psychologique de Charlie}

La théorie de la personnalité qui se concentre sur « l'actualisation de soi » s'intéresse aux individus créatifs. Maslow dans Alwisol (2014, p. 199) aussi a ajouté qu'une personne qui accepte son obligatoire pour sa vie, sera conscient de son potentiel, il sera capable de surmonter les influences données par l'éducation de ses parents, l'éducation à l'école, et aussi les autres pressions. Il a aussi déclaré qu'en effet les humains, sont les bons individus qui ne poussent pas à faire le mal comme les démons. Chaque individu a son besoin, ses compétences et ses tendances génétiques. Parmi ces caractères deviennent les caractéristiques générales des humains, tandis que les autres caractères deviennent les caractéristiques individuelles.

Essentiellement, les besoins, les compétences et les tendances des humains sont bons et ce ne sont pas mauvais. Les caractéristiques de démon qui sont mauvais, destructif, et sévérité sont causés par des frustrations car une personne n'est pas toujours satisfaite à ses besoins, cela est caractère héréditaire (Alwisol, 2014, p. 200). Ce n'est pas tout, Alwisol a aussi ajouté que les humains ont le potentiel de se développer de devenir le meilleur de soi-même, mais c'est possible de changer en même temps l'effort de faire satisfaire ses besoins fondamentaux, et ce n'est pas un caractère héréditaire. Nous pouvons dire que les humains ont le potentiel pour se développer d'être un individu positif, mais il peut changer et il dépende de la satisfaction de ses besoins.

Pour accomplir nous-mêmes ou réaliser «l'actualisation de soi », les humains ont ses certains besoins. Maslow (1987, p. 35) a arrangé une théorie de motivation des humains, dans cette théorie les variétés de besoins humains sont arrangées dans une hiérarchie constituée de cinq niveaux. Les niveaux de la motivation se lient l'un et l'autre, alors un individu recherche d'abord la satisfaction de ses besoins de base physiologiques et après il compléter les besoins aux niveaux supérieurs. 
Maslow ne présente jamais tous les besoins sous forme d'une pyramide, mais dans cette recherche nous avons présenté ces besoins sous forme de pyramide pour que nous puissions voir les hiérarchies de besoins facilement comme le suivant :

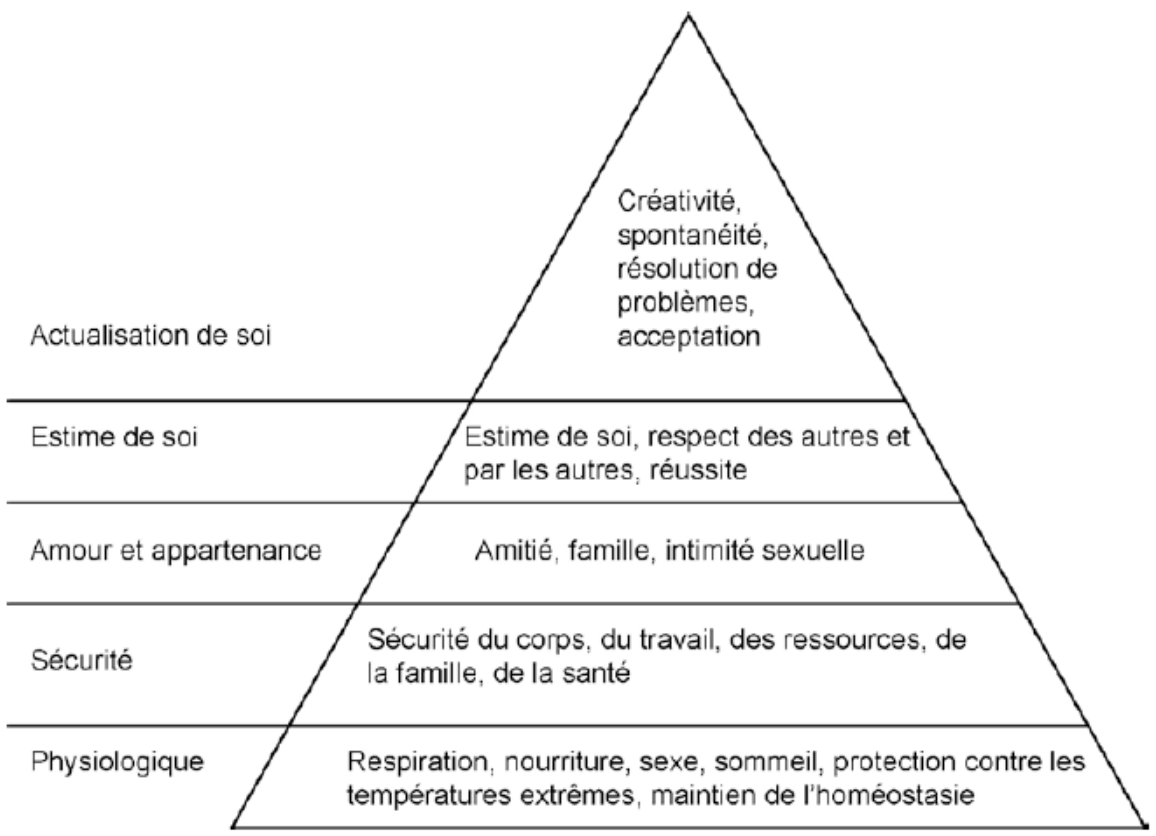

Figure 1 Schéma adapté de la pyramide

Les quatre premiers besoins fondamentaux des individus sont le besoin physiologique, le besoin de sécurité, le besoin d'amour et d'appartenance celui le besoin d'estime de soi. Ces quatre besoins sont les besoins de base. Selon Maslow dans Mehran (2010, p. 40), les besoins physiologiques sont liés au maintien de l'homéostasie de l'organisme. Ce sont les plus évidents de tous les besoins humains : manger, respire, dormir, se protéger contre les températures extrêmes, avoir des rapports sexuels, évacuer les déchets corporels.

Le besoin de sécurité est le deuxième besoin apparu après avoir obtenu la satisfaction physiologique. Il s'agit d'obtenir un degré raisonnable de certitude, d'ordre, de structure et de prévisibilité. Parmi les besoins de sécurité, nous trouvons la sécurité d'une maison, de physique (contre la violence), de la stabilité familiale, la sécurité de l'emploi et des revenus, et aussi de la santé. En plus, les besoins de sécurité sont aussi les besoins physiologiques pour préserver la vie. Le besoin physiologique est une défense temporaire tandis que le besoin de sécurité est une défense à long terme. Dans ce film, la dispute des parents de Charlie qui se passe souvent dirigent leur relation au divorce. Le divorce cause désorganisation dans sa famille et donne Charlie l'insécurité dans sa propre famille. L'autre forme de l'insécurité est montrée comme la maladie de Charlie qui donne l'insécurité de santé. Ajouté par la menace venant de Sarah, Sarah a menacé Charlie qu'elle lui va tuer si elle parle de sa mère. Cette menace provoque l'insécurité contre la violence et donne l'effet psychologique par Charlie. Ce n'est pas tous, Sarah aussi fait terreurs téléphoniques pour déranger Charlie, ça donne l'insécurité de stabilité dans sa vie.

Le troisième besoin est les besoins d'amour et appartenance, il s'agit du besoin d'être intégré et de participer à un group, d'être reconnu et pris en considération, le besoin d'aimer et d'être aimé affectueusement et sexuellement. Un individu va chercher une place dans sa famille et/ou dans son groupe de référence. Le sentiment d'appartenance peut provenir d'un groupe social (religieux, culturel, professionnel, sportif ou même un gang de rue). C'est le besoin essentiel que Charlie ne gagne pas. Commencé par Sarah qui tourne vers les autres amis et vers Vanessa, Charlie ne sent plus aimée et elle n'intégrée plus avec Sarah. Charlie qui s'isole progressivement de tous a causé désintégration avec ses amis. Après avoir être faible à cause de l'insatisfaction de ce besoin, Charlie n'est prise plus en considération venant Sarah. Il est montré dans la scène ou Sarah démantèle le secret personnel de Charlie sur Lucas.

Le dernier des besoins de base est le besoin d'estime de soi. Quand les besoins d'appartenance et d'amour sont raisonnables et satisfaisant, les forces motivationnelles diminuent et ouvrent la voie aux besoins d'estime de soi. A ce niveau, il apparaîtra le besoin de respect de soi comme le désir de compétence, de force personnelle, d'accomplissement, d'indépendance, de liberté, et le fait d'être à la hauteur des situations. Une personne doit avoir la connaissance sur lui-même, soit apprécié, sois capable de faire les taches dans sa vie. 
En même temps il a aussi le besoin d'estime venant des autres, comme le désire d'être respecté par les autres, on trouve le prestige, le statut social, la réputation, la réussite et la reconnaissance. Dans ce film, Charlie n'est pas respecté à cause des harcèlements donné par Sarah. Charlie a vécu toutes la moquerie et l'humiliation qui ne répondent pas à son désire pour d'être respectée et qui mettre mal à sa réputation. En plus, ces terreurs téléphoniques qui lui dérange donnent l'effet a son étude, c'est-à-dire elle ne peut pas obtenir la réussite.

Enfin une fois que tous les autres besoins ont été satisfaits, Maslow déclare que le besoin d'actualisation de soi se met en avant. Ajouté par Mehran (2010, p. 37) qui a dit que l'actualisation de soi peut être considérée comme la nécessité instinctive de l'homme d'atteindre le sommet de son potentiel et de mettre en œuvre l'ensemble de ses capacités. Elle n'a pas besoin de prendre une forme créative et artistique. Un étudiant, une mère, un enseignant peuvent actualiser leurs compétences potentielles et font les choses de leur mieux. Les différentes formes d'actualisation de soi diffèrent d'une personne à l'autre. Selon Maslow (1987, p. xx) des personnes qui réussissent d'actualiser le soi sont personnes saines et matures. Malheureusement, l'insatisfactions les autres besoins précédents ne lui permet pas d'actualiser son soi.

\section{Conclusion}

Nous trouvons les formes du conflit intérieur de Charlie comme le personnage principal de l'œuvre cinématographique "Respire ». Nous avons analysé les données obtenues en utilisant la théorie d'humaniste sur motivation et personnalité. Ce conflit intérieur trouvé est l'insatisfaction des besoins fondamentaux comme le besoin de sécurité, d'amour et d'appartenance et celui d'estime de soi. Puisque ses besoins fondamentaux insatisfaisants, Charlie n'est pas motivé à satisfaire son besoin d'actualisation de soi comme le sommet de la hiérarchie des besoins d'humain.

\section{Références}

Alwisol. (2009). Psikologi Kepribadian. Malang: UMM Press.

Battaglia, M. (2017). Harcèlement scolaire: « Un élève sur dix est concerné. Retrieved from

http://www.lemonde.fr/education/article/2017/11/09/harcelement-scolaire-un-eleve-sur-dix-estconcerne_5212707_1473685.html\#XAvOKzYBsjA8ItkA.99

Maslow, A. (1987). Motivation and Personality. New York: Harper and Row.

Mehran, F. (2010). Psychologie positive et personnalité. Paris: Elsevier Masson. 